\title{
Colloidal silicon nanocrystallites for low-cost solar cell development
}

\section{V. Švrček*}

In this letter a formation of solution based of bulk-heterojunction based on freestanding silicon nanocrystals (Si-ncs) and conjugated ((poly(3-hexylthiophene) (P3HT) polymer is demonstrated. Surfactant free Si-ncs prepared by low-cost electrochemical etching are applied for fabrication of bulk-heterojunction and photo-conductive blends. We show that the optimum blend performance is at $40 \mathrm{wt} \%$ nanocrystallites concentration within the P3HT polymer matrix. Furthermore, we illustrate that solar cell transport properties can be improved by nanosecond laser fragmentation of the nanocrystallites micrograins in ethanol. It argues that the Si-ncs/polymer blend with refine nanocrystaline structure may impact the development of low-cost solar cells by environmental- friendly mean.

Keywords: Silicon; Nanocrystals; Electrochemical etching; Solar cell

Citation: V. Švrček, "Colloidal silicon nanocrystallites for low-cost solar cell development”, Nano-Micro Lett. 1, 40-44 (2009). doi: 10.5101/nml.v1i1.p40-44

Solar cells have become nowadays ever more attractive as an alternative source of green energy [1-3]. A conjugated polymer based solar cells particularly present one perspective choice for the development of solar cells industry on large areas and at low cost [3-5]. Most of the polymer solar cells today rely on the formation of bulk-heterojunction i.e. a interpenetrating network of donor and acceptor material [4-6]. In last decade the fast photo-induced charge transfer from a conjugated polymer to fullerene showed to be very efficient and this system considerably speed up polymer based solar cell development $[7,8]$. Nowadays best polymer based solar cells having a electrical conversion efficiency in the range of 3-8\% [9-12]. However, still vigorous period of research is needed to refine the structure processing and to develop the solar cell fabrication techniques.

One-way to improve the efficiency is blending of polymer with a second inorganic nano-composite. On the other hand, it is accepted in photovoltaic community that to achieve a considerable improvement in solar cell efficiency a new physics as quantum-confined structures have to be applied [13-15]. One of such approach involves so called "hybrid" photovoltaic structures, consisting of blending of organic polymers with inorganic semiconductor nanocrystals (quantum dots), possessing of quantum confinement size effect (e.g. CdS, CdSe, and CdTe) [16]. Better yet, similar to fullerene, the nanocrystal can also serves as an acceptor for electrons [17] or potentially as a donor when it is doped.

Silicon nanocrystals (Si-ncs) are one potential low dimensional nanocomposite that fulfills all criteria for the next generation of solar cell development [13]. It has been demonstrated that it can be also efficiently used for bulk-heterojunction formation when blended with conjugated polymers [17,18]. Natural micro/nano phase-separation between the polymer and the solid state Si-nc get the morphology/structure to obtain photogenerated carrier separation $[17,18]$. In addition, it is expected that Si-ncs with quantum confinement size effect may provide a significant boost in carrier generation efficiency from a phenomenon so called carrier multiplication [19-22]. The principle of the phenomenon is sketched in Fig. 1. Illuminated nanocrystal 
responds to an absorbed photon by producing multiple electron-hole pairs. So far in many types of nanocrystals the phenomenon has been widely reported [20-22]. It has to be stressed higher multiplication rates were reported for low band gap nanocrystals (e.g. CdSe). However, since the Si-ncs possess non-toxicity for the environment, abundance and established silicon based photovoltaic technologies Si-ncs even lower carrier multiplication rate could be more favorable material for solar cell production process at lower cost.

Processing of freestanding Si-ncs allows homogenous dispersion in almost any liquid, polymers included. It enables tuning of nanocrystals concentration in the host matrix very simply and high concentration assuring percolation trajectories for Si-ncs can be simply achieved [17]. Those might directly impact the blend performance. Even more, the interface between the organic and soluble nanocrystal can be controlled by using chemical or physical methods. Such flexibility considerably improves the opto-electronic interaction with host polymer matrix and allows better energy transfer within the system (nanocrystal/polymer). Recently introduced laser fragmentation of silicon micrograins can tune efficiently the surface chemistry and allows closed pack integration of Si-ncs within self organized structures without using any surfactant [23]. It is believed that a nanosecond laser processing of the Si-ncs in liquid and blending with suitable conjugated polymer may considerably impact solar cell performance.

Here we demonstrate a fabrication of bulk heterojunction based on blending of freestanding Si-ncs with ((poly(3hexylthiophene) (P3HT) polymer. Cheap electrochemical etching is applied for preparation of free standing and surfactant free Si-ncs with bright-red photoluminescence (PL) at room temperature. Si-ncs blended with polymer show an improvement in photo-conductivity response and the optimum performance is observed at $\sim 40 \mathrm{wt} \%$ concentration. Further, we show that an improvement of the nanocrystals surface passivation and morphology by nanosecond laser fragmentation processing in liquid media enhances the transport properties of the solar cell device.

In this work the freestanding doped Si-ncs are prepared by electrochemical etching of a silicon wafers. Boron doped wafer with a resistivity of $0.5-0.75 \Omega \cdot \mathrm{cm}$ (p-type, $<100>$ ) was used. The wafers were electrochemically etched in a mixture of hydrofluoric acid with pure ethanol $\left(\mathrm{HF}: \mathrm{C}_{2} \mathrm{H}_{5} \mathrm{OH}=1: 4\right.$, current density $3.2 \mathrm{~mA} / \mathrm{cm}^{2}$ and etching time $60 \mathrm{~min}$ ). After etching the Si-ncs were collected by mechanical scratching and sedimentation [24].

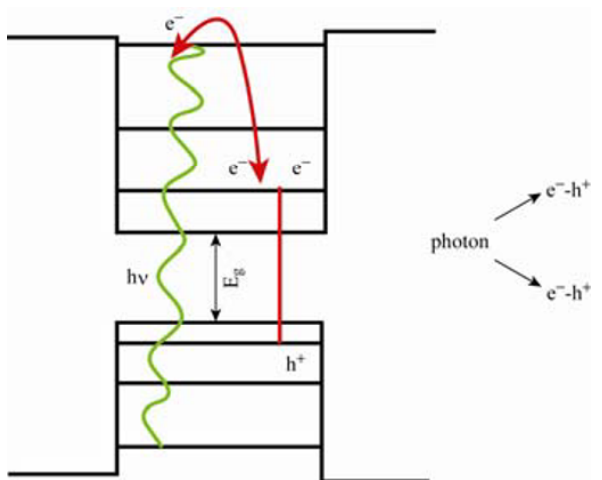

FIG. 1. A schematic sketch of carrier multiplication in excited nanocrystal possessing of quantum confinement size effect.

For blend formation a commercially available (ALDRICH) polymer (poly(3-hexylthiophene) (P3HT) was used. Blends were prepared by dissolving $10 \mathrm{mg}$ of polymer in $10 \mathrm{~g}$ of chlorobenzene. Then freestanding Si-ncs were introduced at different concentrations. In order to study photoconductivity measurement $300 \mathrm{~nm}$ thin films were spun cast on a glass covered by interdigitated platinum contacts. The interdigitated contacts consisted of 20 fingers, with a length of $6 \mathrm{~mm}$ and a width of $200 \mu \mathrm{m}$, separated by $200 \mu \mathrm{m}$. At the same conditions another set of samples just with pure polymers (P3HT) were fabricated for comparison. After processing all samples were dried at $140^{\circ} \mathrm{C}$ for $30 \mathrm{~min}$ in vacuum.

At the $40 \mathrm{wt} \%$ concentration of Si-ncs the solar cell device has been made by following way. Glass substrates with an indium tin oxide electrode were used for solar cells fabrications. In order to smooth the ITO surface the poly(3,4-ethylenedioxythiophene):poly(styrene sulfonic acid (PEDOT: PSS) was deposited by spin coating (3000 rpm, dried at $\left.135^{\circ} \mathrm{C}\right)$. Subsequently, the Si-ncs/P3HT solution was spin-coated on the PEDOT:PSS/ITO substrate. Then, an alumina top electrode of $100 \mathrm{~nm}$ was deposited on top of the active layer by vacuum

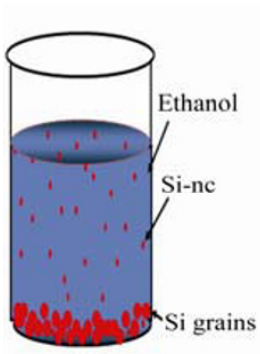

(a)

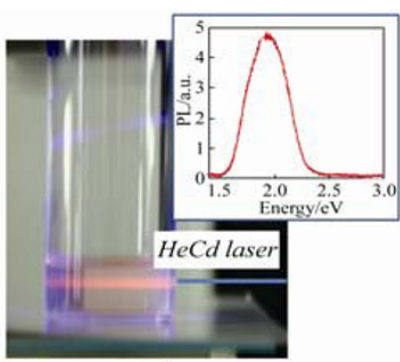

(b)
FIG. 2. (a) The sketch of the experiment to collect the electrochemically etched Si-ncs prepared by mechanical removal of porous silicon wafers. (b) Typical photos of the colloidal solution after micrograins sedimentation in ethanol. The colloid is excited with the $\mathrm{He}: \mathrm{Cd} \mathrm{cw}$ laser at $325 \mathrm{~nm}$ wavelength. Inset shows corresponding photoluminescence (PL) spectra of harvested Si-ncs taken at room temperature $(\mathrm{T}=300 \mathrm{~K})$. 
evaporation. The active area of the cell was $4 \mathrm{~mm}^{2}$.

It has to be noted that the PL, conductivity and the photoconductivity measurements were conducted in air atmosphere and ambient conditions. To measure PL the samples, an $\mathrm{HeCd}$ laser with a wavelength of $325 \mathrm{~nm}(3.82 \mathrm{eV})$ was used. In order to measure $\mathrm{I}-\mathrm{V}$ characteristics a voltage from a regulated DC power supply was applied and the resulting current was measured with a KEITHLEY 6430. For photoconductivity measurement a white light of $1.5 \mathrm{AM}$ was used. Spectrally resolved photocurrent of the solar cell was measured under illumination with monochromatic light from a Xe lamp. In all cases the irradiation intensities were calibrated by a standard a-Si solar cell.

Figure 2(a) shows a schematic sketch for a collection of fine silicon crystallites dispersed in ethanol. After a scratching procedure [19] and dispersion of powder in ethanol the largest micrograins get sediment on the bottom of the glassware. The finest used for further blend fabrication are harvested from the upper liquid after evaporation of ethanol. Figure 2(b) shows typical photo of the upper solution after sedimentation for 4 hours. When the largest agglomerates sediment the upper solution becomes transparent that suggests that only the smallest nanocrystals remained. In order to test the presence of Si-ncs bright room temperature PL feature is used to witness them. When the laser beam is shined through the solution red PL is observed (Fig. 2(b)). This proofs that we deal with the Si-ncs with quite good quality. Low concentration defects (surface or bulk) do not hinder emission significantly. Further structural analysis [25] witnessed the presence Si-ncs with diamond-like structure with average size about $3 \mathrm{~nm}$. Corresponding PL spectra is shown in inset of the Fig. 2(b). The PL spectrum is rather broad with maximum centered at $2 \mathrm{eV}$. As reported elsewhere the PL in Si-ncs with grain size of less than $5 \mathrm{~nm}$ originate mostly from quantum confinement effects [25-27]. Similar arguments could be used to describe luminescence properties of our sample. Therefore one can

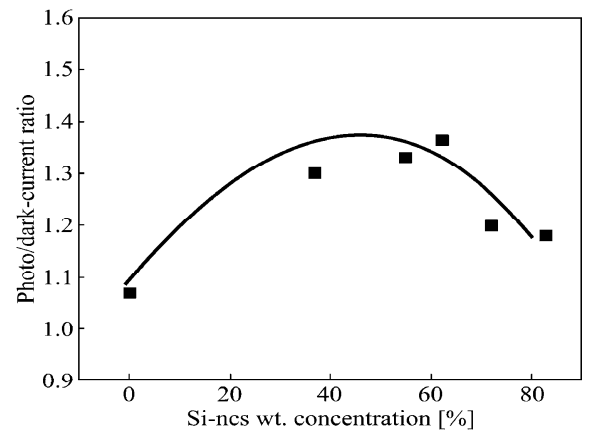

FIG. 3. Photo-/dark-conductivity ratio as a function of Si-ncs concentration in P3HT polymer matrix. expected that a significant spectrum broadening could be ascribed to large nanocrystal size distribution.

Recently it has been demonstrated that such a way prepared Si-ncs blended with conjugated polymer form a bulk-heterojunction [17,28]. As the freestanding Si-ncs concentration within the polymer can be easily tuned, the photoconductivity response of the blend at different nanocrystalites concentration has been optimized. It has been observed that the photo- and dark-conductivity ratio varied as a function of Si-ncs concentration (Fig. 3). As the Si-ncs concentration is increased also the ratio between photo and dark increases and reaching maximum at around $40 \mathrm{wt} \%$. After reaching the peak the ratio again decreased. It has to be noted that the photoexcitation in silicon nanocrystals is fundamentally different from those in polymers or organics nanoparticles. Particular, whereas light absorption in Si-ncs (quantum dot) results to the direct generation of mobile charged carriers, however, the higher (more than 10 times) light absorption in polymer leads to the generation of excitons [17]. The exciton in polymer is associated with binding energy in excess of $0.2 \mathrm{eV}$. Therefore the exciton and the charged carrier transport in higher absorbing polymer [28] is the heart of the photocarrier generation of the blend. As a result an excitionic energy transfer might be an efficient mechanism to pump Si-ncs through the excitation of conjugated polymers [17]. We stress that the exciton is charge-neutral and is transported due to the diffusion process only. Contrary to that a charged carrier is transported by both diffusion and/or drift in the built-in electric fields. In our case field results from difference in work function of P3HT polymer and ionization potential of the nanocrystal that
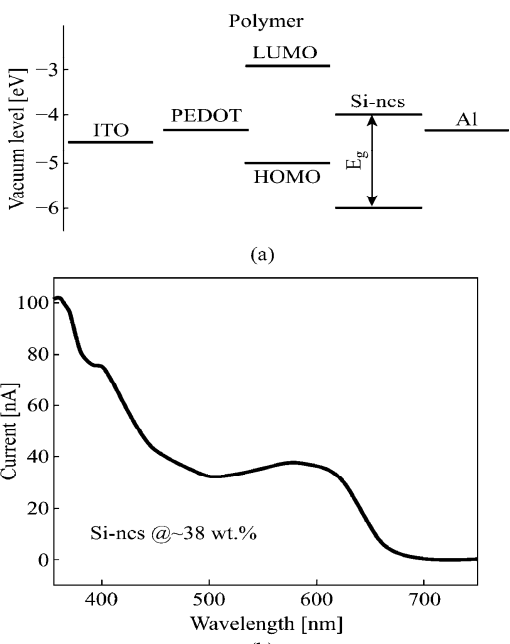

(b)

FIG. 4. (a) Energy level band diagram of silicon nanocrystals (Si-ncs) and conjugated polymer based solar cell. (b) Photocurrent generation as a function of illumination wavelength for the P3HT bulk-heterojunction solar cells containing of Si-ncs prepared by electrochemical etching at $38 \mathrm{wt}$. $\%$. 
dissociates photogenerated excitons [17]. When the concentration is further augmented, most likely the P3HT lamella-like structure become more perturbed, which results in a hole mobility decrease [29]. Those perturbations then limit carrier transport and photoconductivity response of the blend at higher Si-ncs concentrations as $40 \mathrm{wt} \%$.

Furthermore, we have fabricated solar cell based on the blend with silicon nanocrystallites concentration around 40 $w t \%$. This allowed as to test the blend properties in sandwiched device-like configuration Fig. 4(a) shows energy level diagram of Si-ncs conjugated polymer based hybrid solar cell (ITO/PEDOT:PSS/(Si-ncs/P3HT)/Al). The PEDOT:PSS is used as the hole collector in this structure and alumina as the electron. While the polymer band structure is well known (having the highest at $(\sim 5 \mathrm{eV})$ and lowest unoccupied molecular orbital (LUMO) level at $\sim 2.9 \mathrm{eV}$ ) the ionization potential of the Si-ncs is not well determined yet. Meanwhile it is assumed that it does not differ significantly to bulk silicon and adjust to band gap of the Si-ncs [17]. It is considered that band offset decreases due to quantum confinement and wide band gap of Si-ncs. Red photoluminescent Si-ncs having optical band gap around $\sim 2 \mathrm{eV}$ and work function being around $\sim 4.1 \mathrm{eV}$ that can be favorably aligned with P3HT polymer. Then, different electron affinity and ionization potential between the nanocrystal and polymer provide a driving force for dissociating the excitons. It has to be noted that similar to parallel configuration as the concentration of Si-ncs is increased also the generation of the photocurrent in solar cell is enhanced by reaching maximum around $\sim 40 \mathrm{wt} \%$.

Figure 4(b) represents photocurrent generation as a function of wavelength for the blends with at $38 \mathrm{wt} \%$ ).

Figure 5(a) represents corresponding I-V characteristics of hybrid solar cell. Red line is recorded under AM1.5 illumination and black line I-V characteristic taken in dark. Since $\mathrm{V}_{\mathrm{OC}}$ is relatively high the generated photocurrent is weak. This is due to the multiple factors that have to be taken into account. In order to improve the photocurrent generation the oxidation and formation of oxide shell has to be avoided during the multiple technological processes. Otherwise, oxidized shell hinders overall transport properties and deteriorates the fill factor of the solar cell. Even more, inorganic oxides especially under UV irradiation can catalyze the photodegradation in polymers due to the reversibly depletion of oxygen under illumination [2]. These processes then compete with the charge separation and decreases overall performance of solar cell.

Nanocrystal oxidation can be efficiently overcome by conducting the fabrication process in inert atmosphere or
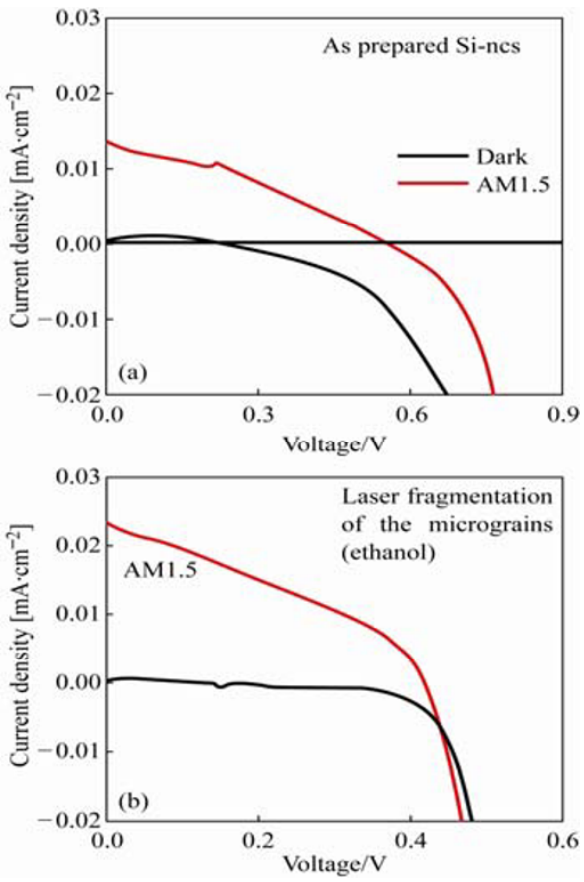

FIG. 5. (a) Corresponding I-V characteristic of Si-ncs (38 wt. \%) embedded in P3HT polymer. (b) I-V characteristic of the device made from Si-ncs micrograins fragmented in ethanol and blended with P3HT at $\sim 38$. wt.\% concentration. Red lines are recorded under AM1.5 illumination and the black curves in dark.

preparing Si-ncs in less oxidazing liquids by newly developed techniques (i.e. laser processing). In order to show the potential of laser processing we elaborated Si-ncs micrograins in ethanol by nanosecond laser fragmentation. Figure 5(b) presents I-V characteristics of the device made from such defragmented Si-ncs micrograins and blended with P3HT (at same weight concentration $(\sim 38 \%))$. Red lines are recorded under AM1.5 illumination and the black ones in dark. We could clearly observe that defragmentation increased the generation of the photocurrent in the solar cell and an improvement in fill factor has been recorded as well. This is mostly due to the formation of thinner oxide shell around nanocrystals during the fragmentation process in ethanol compare to exposure to the air. However, due to the fabrication of larger sized nanocrystallites a decrease in energy band gap occurred. This could be beneficial factor for better matching with solar spectra, however balance between the fragmentation conditions and Si-ncs size has to be find. Otherwise decrease in band gap reduces the open circuit voltage $\left(\mathrm{V}_{\mathrm{OC}}\right)$. Unfortunately, since in this work we focalized primary to show the principles, the structure morphology was not optimized yet. Therefore, further structural changes can occur when the fragmentation is performed in less oxidizing liquids at optimal fragmentation conditions. It has to be noticed that the fragmentation in liquid at the same time allows tuning of nanocrystallites surface properties without any 
additional surfactant [22], which might considerably improve the solar cell properties.

In conclusion, we fabricated by electrochemical etching top-down approach freestanding and surfactant-free photoluminescent silicon nanocrystals (Si-ncs). We showed that the blending with poly(3-hexylthiophene (P3HT) conjugated polymer allowed formation of solution based bulkheterojunction. The different electron affinity and ionization potential between the Si-nc and P3HT dissociates excitons and allowed photocurrent generation. We demonstrated that the largest rate between photo- and dark-conductivity has been achieved at Si-ncs concentration around $\sim 40 \mathrm{wt} \%$. Furthermore, we showed a strategy for an improvement of photocurrent generation in solar cells by applying of Si-ncs prepared by nanosecond laser fragmentation in ethanol.

This work was also partially supported by a NEDO project.

Received 30 November 2009; accepted 15 December 2009; published online 20 December 2009.

\section{References}

1. M. C. Scharber, D. Mühlbacher, M. Koppe, P. Denk, C. Waldauf, A. J. Heeger and C. J. Brabec, Adv. Mater. 18, 789 (2006). doi:10.1002/adma.200501717

2. M. Jørgensen, K. Norrman and F. C. Krebs, Sol. Energy Mater. Sol. Cells, 92, 686 (2008). doi:10.1016/j.solmat. 2008.01.005

3. R. A. J. Janssen, J. C. Hummelen and N. S. Sariciftci, MRS Bull. 30, 33 (2005).

4. M. Lira-Cantu, K. Norrman, J. W. Andreasen and F. C. Krebs, Chem. Mater. 18, 5684 (2006). doi:10.1021/ cm061429d

5. K. M. Coakley and M. D. McGehee, Chem. Mater. 16, 4533 (2004). doi:10.1021/cm049654n

6. I. Alexandrou, E. Lioudakis, D. Delaportas, et al. Nanoscale Res. Lett. 4, 635 (2009). doi:10.1007/s11671009-9287-9

7. C. J. Brabec, Sol. Energy Mater. Sol. Cells 83, 273 (2004). doi:10.1016/j.solmat.2004.02.030

8. C. J. Brabec and V. Dyakonov, in Organic Photovoltaics: Concepts and Realization, edited by C. J. Brabec, V. Dykonov, J. Parisi and N. S. Sariciftci (Springer-Verg, Berlin, 2003).

9. F. Padinger, R. S. Rittberger and N. S. Sariciftci, Adv. Funct. Mater. 13, 18 (2003). doi:10.1002/adfm.20039 $\underline{0011}$
10. P. Peumans and S. R. Forrest, Appl. Phys. Lett. 79, 126 (2001). doi:10.1063/1.1384001

11. M. M. Wienk et al. Angew. Chem. Int. Ed. 42, 3371 (2003). doi:10.1002/anie.200351647

12. S. H. Park et al. Nature Photonics 3297 (2009).

13. M. A. Green, Third Generation Photovoltaics, Bridge Printery, Sydney (2001).

14. A. Luque and A. Marti, Phys. Rev. Lett. 78, 5014 (1997). doi:10.1103/PhysRevLett.78.5014

15. T. Trupke, M. A. Green and P. Wurfel, J. Appl. Phys. 92, 4117 (2002). doi:10.1063/1.1505677

16. W. U. Huynh, X. Peng and P. Alivisatos, Adv. Mater. 11, 923 (1999).

17. V. Švrček, H. Fujiwara and M. Kondo, Appl. Phys. Lett. 92, 143301 (2008). doi:10.1063/1.2905269

18. C. Liu, A. Holman and U. Kortshagen, Nano Lett. 9, 449 (2009). doi:10.1021/nl8034338

19. A. J. Nozik and Philos. Trans. R. Soc. London Ser. A 295, 453 (1980). doi:10.1098/rsta.1980.0141

20. A. J. Nozik, Physica E 14, 115 (2002). doi:10.1016 /S1386- 9477(02)00374-0

21. R. Schaller and V. Klimov, Phys. Rev. Lett. 92, 186601 (2004). doi:10.1103/PhysRevLett.92.186601

22. M. C. Beard, K. P. Knutsen, P. Yu, J. M. Luther, Q. Song, W. K. Metzger, R. Ellingson and A. J. Nozik, Nano Lett. 7, 2506 (2007). doi:10.1021/n10714861

23. V. Švrček, D. Mariotti, K. Kalia and M. Kondo, Chem. Phys. Lett. 478, 224 (2009). doi:10.1016/j.cplett. $\underline{2009.07 .090}$

24. V. Švrček, A. Slaoui and J. C. Muller, J. Appl. Phys. 95, 3158 (2004). doi:10.1063/1.1649817

25. V. Švrček, H. Fujiwara and M. Kondo, Solar Ene. Mate. Solar Cells 93,774 (2009). doi:10.1016/j.solmat.20 08.09 .033

26. M. L. Brongersma, A. Polman, K. S. Min, E. Boer, T. Tambo and H. A. Atwater, Appl. Phys. Lett. 72, 2577 (1998). doi:10.1063/1.121423

27. M. V. Wolkin, J. Jorne, P.M. Fauchet, G. Allan and C. Delerue, Phys. Rev. Lett. 82, 197 (1999). doi:10.1103/ PhysRevLett.82.197

28. V. Švrček, H. Fujiwara and M. Kondo Acta Mater. 57, 5986 (2009). doi:10.1016/j.actamat.2009.08.025

29. X. Yang, J. Loos, S. C. Veenstra, W. J. H. Verhees, M. M. Wienk, J. M. Kroon, M. A. J. Michels and R. A. J. Janssen, Nano Lett. 5, 579 (2005). doi:10.1021/n1048120i 